\section{$\underset{\substack{\text { hommes } \\ \text { \& migrations }}}{ }$}

\section{Hommes \& migrations}

Revue française de référence sur les dynamiques

migratoires

$1326 \mid 2019$

Londres et ses migrations

\title{
Causes des migrations et conséquences du Brexit sur le marché du travail
}

\section{Zovanga Kone et Carlos Vargas - Silva}

Traducteur : Vanessa Kientz

\section{(2) OpenEdition}

\section{Journals}

\section{Édition électronique}

URL : https://journals.openedition.org/hommesmigrations/9613

DOI : 10.4000/hommesmigrations.9613

ISSN : 2262-3353

\section{Éditeur}

Musée national de l'histoire de l'immigration

Édition imprimée

Date de publication : 1 juillet 2019

Pagination : 43-47

ISBN : 978-2-919040-46-9

ISSN : $1142-852 X$

\section{Référence électronique}

Zovanga Kone et Carlos Vargas - Silva, « Causes des migrations et conséquences du Brexit sur le marché du travail », Hommes \& migrations [En ligne], 1326 | 2019, mis en ligne le 01 janvier 2022, consulté le 14 janvier 2022. URL : http://journals.openedition.org/hommesmigrations/9613 ; DOI https://doi.org/10.4000/hommesmigrations.9613 


\section{Causes des migrations et conséquences du Brexit sur le marché} du travail

Zovanga Kone, Research Officer, Centre on Migration, Policy and Society, University of Oxford, et Carlos Vargas-Silva, Senior researcher, Centre on Migration, Policy and Society, University of Oxford.

La sortie du Royaume-Uni de l'Union européenne questionne la politique migratoire britannique, notamment en direction des ressortissants de l'UE. Afin de prévoir les conséquences d'une modification de l'accès de ces ressortissants sur l'immigration de main-d'œuvre, et donc sur le marché du travail outre-Manche, le gouvernement britannique a produit, fin 2018, un Livre blanc précisant le nouveau régime d'immigration envisagé. Sa principale proposition concerne l'application des règles britanniques en matière d'immigration à tous les immigrants, qu'ils soient ou non, d'origine européenne.

$\underline{\mathbf{L}}$ une des conséquences, pour le RoyaumeUni, du départ de l'Union européenne (UE), avec la fin de la liberté de circulation, est la nécessité de repenser le droit des ressortissants de l'UE à vivre et à travailler dans ce pays. Dans ce contexte, la question essentielle est de savoir quelles sont les possibles implications d'une modification de l'accès des ressortissants de l'UE au Royaume-Uni et à son marché du travail? Pour répondre à cette question, il est nécessaire d'examiner les caractéristiques de leur emploi au RoyaumeUni. En principe, cela devrait être aisé au vu du nombre d'articles universitaires consacrés au sujet. Mais, en réalité, leur examen approfondi fait apparaître une lacune importante: la grande hétérogénéité de la main-d'œuvre de l'UE n'est pas prise en compte. Deux catégories de ressortissants sont principalement distinguées, ceux provenant de pays qui étaient membres de l'UE avant 2004 (UE-14) et ceux provenant de nouveaux États membres de l'UE (NEM)'. Cependant, au sein même de ces deux groupes, les raisons poussant les individus à immigrer au Royaume-Uni varient considérablement: trouver un

1. Stephen Drinkwater, John Eade, Michal Garapich, «Poles apart? EU enlargement and the labour market outcomes of immigrants in the United Kingdom », in International Migration, vol. 47, $n^{\circ} 1,2009$, pp. 161-190. 
emploi, étudier, rejoindre ou accompagner des membres de leur famille, etc. Cette hétérogénéité des motifs d'immigration des ressortissants de l'UE a été largement ignorée par la littérature. Il s'agit pourtant d'un point non négligeable, car toute nouvelle règle d'immigration est susceptible d'établir des distinctions fondées sur la voie d'entrée et le motif de la migration. En d'autres termes, il est probable que des règles différentes s'appliqueront à ceux qui viendront pour travailler, rejoindre des membres de leur famille ou étudier².

\section{Les motifs d'installation des ressortissants de l'UE au Royaume-Uni}

En 2017, 3,8 millions de ressortissants de l'UE vivaient au Royaume-Uni (sans compter les ressortissants britanniques), dont 1,7 million de l'UE-14 et 2,1 millions des NEM. $8 \%$ d'entre eux sont effectivement nés au Royaume-Uni. Parmi les ressortissants de l'UE nés à l'étranger, $46 \%$ ont émigré au Royaume-Uni pour des raisons économiques, $27 \%$ pour des raisons familiales, $10 \%$ pour des raisons d'études et $8 \%$ pour d'autres raisons (graphique 1). Alors que la migration professionnelle est au centre des discussions sur le Brexit et les relations futures entre le Royaume-Uni et l'UE, on constate que la moitié des ressortissants de l'UE vivant au Royaume-Uni a migré vers ce pays pour d'autres raisons.

Il est également intéressant de comparer ces deux groupes de ressortissants de l'UE UE : Ue-14 et

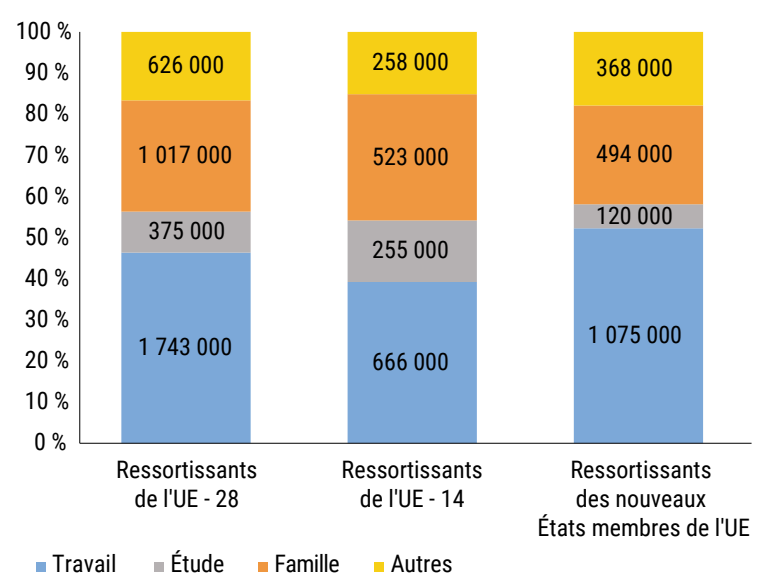

- Figure 1 - Motif initial de migration des ressortissants de I'UE résidant au Royaume-Uni en 2017

Note : «Autres » inclut ceux qui ont initialement migré pour une visite ou pour un motif inconnu, ainsi que les personnes nées au Royaume-Uni.
NEM. En premier lieu, la part de ceux qui ont émigré pour des raisons d'études est deux fois plus importante parmi les ressortissants de l'UE-14 que parmi les ressortissants des NEM. Toutefois, il faut souligner que les raisons de la migration discutées ici ne concernent pas à l'activité principale actuelle. Par exemple, une grande partie des personnes qui sont venues au Royaume-Uni pour étudier ont décidé d'y rester après avoir terminé leurs études. En second lieu, la part de ceux qui ont émigré pour des raisons économiques est $50 \%$ plus élevée parmi les ressortissants des NEM que parmi les ressortissants de l'UE-14. De fait, les ressortissants des NEM représentent trois cinquièmes de l'ensemble des ressortissants de l'UE qui ont émigré pour des raisons économiques, mais seulement un tiers de ceux qui ont émigré pour raison d'études. De même, le nombre et la concentration des personnes qui ont émigré pour raison familiale varient d'un groupe à l'autre.

Les différences observées entre les groupes de l'UE-14 et des NEM reflètent le poids important de certains groupes nationaux dans chaque catégorie. À titre d'exemple, les ressortissants polonais et roumains représentent par exemple plus des deux cinquièmes de tous ceux qui ont émigré pour raison économique. Les ressortissants français et italiens représentent un quart de tous ceux qui ont émigré pour raison d'études. Les ressortissants polonais représentent un quart de tous ceux qui ont émigré pour raison familiale.

\section{Les implications économiques des motifs d'entrée sur le territoire britannique}

Nous abordons ici la question des personnes en âge de travailler (16-64 ans) dans le but d'étudier les caractéristiques de l'emploi entre ressortissants de l'UE

2. Cet article s'appuie sur les recherches menées dans le cadre du projet REMINDER (Role of European Mobility and its Impacts in Narratives, Debates and EU Reforms), lequel s'étend sur un cycle de trois années et porte sur les facteurs déterminants de la libre circulation dans l'UE et leurs conséquences. Pour plus de détails, voir le site : https://www.reminder-project.eu/. Les données traitées dans cet article proviennent d'une version en accès sécurisé du UK Labor Force Survey de 2017 produit par I'ONS (Office for National Statistics) et fournie par le Service des données du Royaume Uni. Le traitement de ces données, qu'il s'agisse d'analyse ou d'interprétation, n'engage donc dans cet article ni la responsabilité de I'ONS, ni celle du service de données sécurisées des Archives de données du Royaume-Uni. Nous remercions chaleureusement Madeleine Sumption pour ses précieux commentaires sur l'article. 


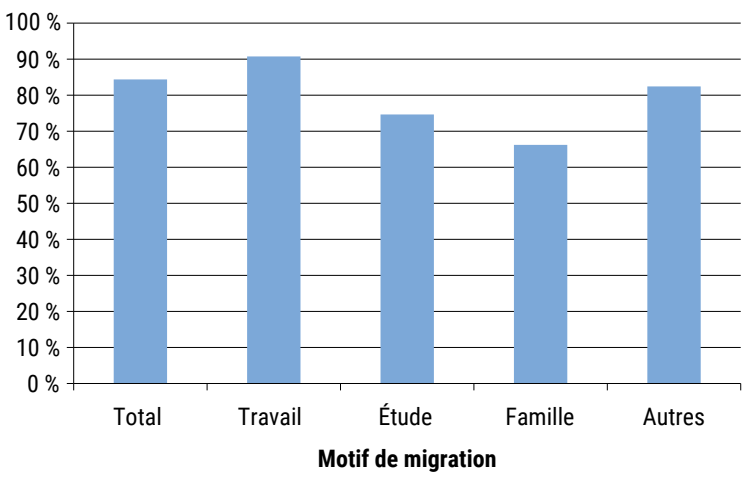

- Figure 2 - Taux d'emploi des ressortissants de l'UE au Royaume-Uni selon le motif de migration, en 2017 (en \%)

ayant migré, quelle qu'en soit la raison. Commençons par le taux d'emploi, soit le nombre de personnes ayant un emploi divisé par le nombre de personnes en âge de travailler. Il n'est pas surprenant de constater que les ressortissants de l'UE qui ont émigré pour raison économique ont le taux d'emploi le plus élevé (supérieur à 90 \%). Mais le taux d'emploi est également élevé dans les autres catégories de ressortissants de l'UE. On observe le taux d'emploi le plus faible parmi les migrants familiaux, à hauteur de $66 \%$. Toutefois, même dans ce cas, il reste supérieur à celui des ressortissants de pays tiers (un peu plus de $60 \%$ ) $^{3}$.

Le graphique ci-dessus suggère que le motif d'admission pourrait expliquer les différents taux d'activité des ressortissants de l'UE. Il est également possible que ce motif ait des implications sur le type d'emploi occupé. Si l'on examine la répartition par profession à l'aide d'une classification des emplois par niveau de compétence ${ }^{4}$, les professions hautement qualifiées (ingénieurs, pharmaciens, professeurs d'université, etc.) sont suivies par les professions moyennement qualifiées supérieures (agents paramédicaux, policiers, moniteurs de fitness, etc.), puis les professions peu qualifiées et les moins qualifiées (travailleurs agricoles, agents d'entretien, opérateurs machine, etc.).

La majorité des personnes occupe des emplois appartenant à cette troisième et dernière catégorie. Elle représente environ un dixième de l'ensemble des personnes exerçant ces professions, soit environ deux fois plus que la proportion de ceux qui exercent des professions exigeant des qualifications plus élevées. Ainsi, les ressortissants de l'UE représentent une part considérable de la main-d'œuvre dans les professions peu qualifiées.

Nous remarquons qu'il existe un fort contraste dans la répartition des professions entre les ressortissants des pays de l'UE-14 et ceux des NEM, même lorsque le motif d'admission est identique. Notons dans un premier temps que indépendamment du motif, les professions moyennement, peu ou les moins qualifiées représentent la plus grande part des emplois occupés par les ressortissants des pays qui ont rejoint l'UE à partir de 2004. Dans ce groupe, ceux qui ont émigré pour motif familial ont la plus forte probabilité d'être employés à ces niveaux de compétence, contrairement à ceux qui ont émigré pour motif d'études. En revanche, la répartition par profession des ressortissants de l'UE originaires de pays qui étaient membres de l'UE avant 2004 est plus équilibrée quant au motif d'admission, bien que ceux qui ont émigré pour motif d'études soient les plus susceptibles d'exercer une profession hautement qualifiée. Enfin, le graphique 3 montre que la migration pour raison familiale est associée aux différences les plus notables entre les ressortissants de l'UE-14 et ceux des NEM.

D'autres études précisant davantage les catégories professionnelles mettent en évidence des concentrations plus importantes de ressortissants de l'UE dans certains emplois et certaines régions. Par exemple, les estimations fondées sur les formulaires des déclarations d'impôts en 2016-2017

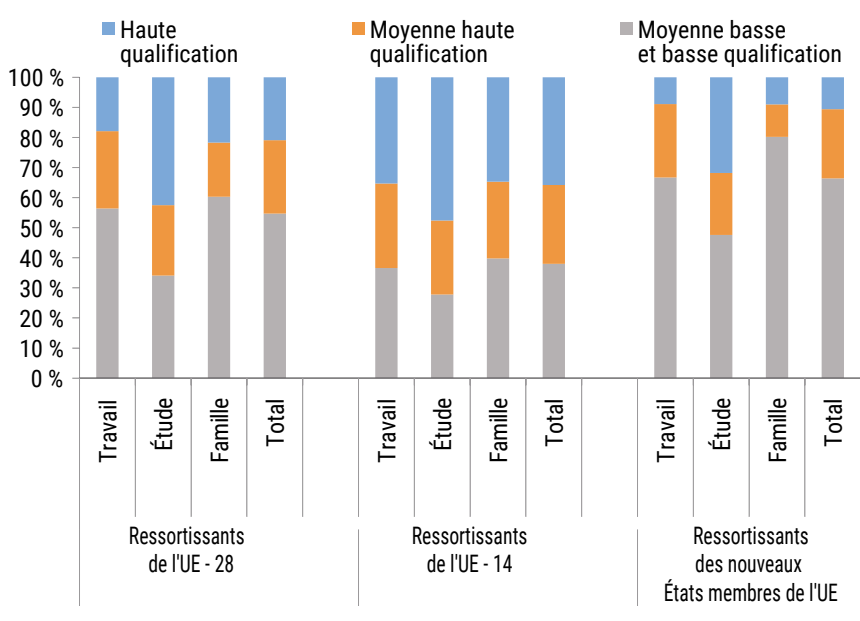

Figure 3 - Répartition par profession selon le motif de migration, 2017 (en \%)

3. UK and Non-UK People in the Labour Market, Londres, ONS, août 2017.

4. SOC2010 (volume 1) : Structure and Descriptions of Unit Groups, Londres, ONS, 2010. Disponible en ligne : https://www.ons.gov. uk/methodology/classificationsandstandards/ standardoccupationalclassificationsoc/soc2010/ soc2010volume1structureanddescriptionsofunitgroups 
suggèrent qu'il y avait plus de ressortissants des NEM travaillant dans le secteur du bâtiment que de ressortissants britanniques, à Londres. Il apparaît, par ailleurs, que les ressortissants de l'UE représentent également la majorité des travailleurs dans les entrepôts dans certaines régions. Leur place est alors essentielle ${ }^{5}$.

\section{Les répercussions sur la politique d'immigration après le Brexit}

Les résultats ci-dessus auront des conséquences sur la politique d'immigration après le Brexit pour les ressortissants de l'UE. Il y a eu, tout d'abord, de nombreux débats au sujet de la politique optimale d'immigration de main-d'œuvre au Royaume-Uni6. La réflexion sur les autres voies d'entrée après le Brexit a été déconnectée des questions liées au marché du travail et à une potentielle pénurie de compétences. La focalisation de la politique migratoire sur le motif professionnel est trop étroite. En effet, une part substantielle des ressortissants de l'UE ont émigré pour un autre motif initial que l'emploi.

Deuxièmement, notre point de vue s'étend au-delà des voies officielles de migration de maind'œuvre. De nombreux facteurs resteront à déterminer au cours des prochaines années, dont les implications seront majeures pour les travailleurs européens. Par exemple, la plupart des ressortissants de l'UE paient les mêmes frais de scolarité que les étudiants britanniques tout au long de leurs études. Les étudiants de l'UE pourront-ils encore y accéder sous les mêmes conditions (frais de scolarité, financement et visa)? Pourront-ils rester et travailler dans le pays après avoir terminé leurs études? Sous quelles conditions? Étant donné que le motif d'études conduit majoritairement à un emploi hautement qualifié, les politiques conçues pour donner la priorité aux migrations de travailleurs hautement qualifiés (comme le soutient actuellement le gouvernement britannique) devraient tenir compte de ce motif et permettre une transition vers le marché du travail. Si les frais de scolarité augmentent et que l'accès au marché du travail devient plus difficile, un effet secondaire, au-delà de l'attraction du Royaume-Uni pour les études, est que le niveau de compétences des travailleurs européens pourrait s'en trouver réduit.

De même, à quelles exigences les personnes qui émigrent de l'UE pour rejoindre leur famille devraientelles répondre? Actuellement, le Royaume-Uni exige un revenu minimum pour le parrainage de visas des conjoints non ressortissants de l'UE?. Les ressortissants de l'UE sont actuellement exemptés de ce seuil de revenu en vertu des règles régissant la libre circulation. Cela pourrait-il changer à l'avenir et de quelle manière?

Pendant longtemps, le gouvernement britannique a évité de préciser quel régime d'immigration remplacerait le régime actuel. Ce fut fait, bien qu'avec un certain retard avec la publication du Livre blanc. Ce document de politique générale a été produit par le gouvernement britannique en décembre 2018 pour proposer les axes d'une législation future. Il contient des informations pertinentes pour les questions examinées ci-dessus ${ }^{8}$. Dans l'ensemble, bon nombre de recommandations émanent du Migration Advisory Committee (MAC), un organe indépendant d'experts chargés de conseiller le gouvernement britannique sur les questions d'immigration. Avant la publication du Livre blanc, en septembre 2018, le MAC avait également publié un rapport sur l'immigration en provenance de l'Espace économique européen (EEE) au Royaume-Uni. L'EEE regroupe les pays de l'UE et les pays membres de l'Association européenne de libre-échange. Nous en avons utilisé les statistiques dans la section précédente.

La principale proposition du Livre blanc est que la libre circulation prendra fin et que «les règles britanniques en matière d'immigration s'appliqueront de la même manière aux migrants européens et non européens» après le Brexit. En d'autres termes, il y aura un système unique, au lieu d'un système qui différencie les individus en fonction de leur pays ou de leur nationalité. Le gouvernement britannique le qualifie de "système d'immigration fondé sur les compétences». Il est prévu qu'il commence à fonctionner à partir de la fin de la «période transitoire» après le Brexit, au cours de laquelle le Royaume-Uni

5. EEA Migration in the UK: Final Report, Migration Advisory Committee, 2018. Disponible en ligne : https://assets.publishing service.gov.uk/government/uploads/system/uploads/ attachment_data/file/741926/Final_EEA_report.PDF

6. Madeleine Sumption, Mariña Fernández-Reino, Exploiting the Opportunity? Low-Skilled Work Migration After Brexit, Migration Observatory Report, 2018.

7. Madeleine Sumption, Carlos Vargas-Silva, «Love is not all you need : Income requirement for visa sponsorship of foreign family members », in Journal of Economics, Race and Policy, vol. 2, n 1-2, 2019, p. 62.

8. Le Livre blanc The UK's Future Skills-Based Immigration System est disponible en ligne : https://www.gov.uk/government/ publications/the-uks-future-skills-based-immigration-system 
restera temporairement dans le marché intérieur européen et dans l'union douanière. Durant cette période, le Royaume-Uni et l'UE négocieraient leurs relations futures, notamment commerciales.

Le Livre blanc fait spécifiquement référence aux migrations pour études et pour raison familiale. Dans le premier cas, le document indique clairement que les étudiants étrangers, européens ou non, devront obtenir une autorisation avant de se rendre au Royaume-Uni. Ceux qui terminent leurs études pourront rester un certain temps pour travailler. En particulier, ceux qui obtiennent une licence et un master dans les universités britanniques bénéficieront d'une période de six mois à l'issue de l'obtention de leur diplôme, pendant laquelle ils pourront travailler sans restriction. Elle sera d'une année complète pour les titulaires d'un doctorat. En outre, ceux qui étudient dans des établissements britanniques pourront demander à passer par la voie des travailleurs qualifiés jusqu'à trois mois avant la fin de leurs études. Cette demande pourra être faite pendant deux ans après la fin de leurs études, de l'extérieur du Royaume-Uni. Globalement, il s'agit d'un système plus généreux à l'égard des ressortissants de pays hors de l'UE que le système actuel, mais il représente un changement majeur pour les ressortissants de l'UE. De plus, ces dispositions demeurent moins généreuses que celles offertes aux étudiants étrangers dans des pays comme l'Australie ou le Canada.

Dans le cas d'une migration familiale, le Livre blanc indique que le Brexit «permettra au RoyaumeUni de mettre en place des règles équitables et cohérentes pour tous». Contrairement au motif d'études, plus souple pour les ressortissants de pays tiers que pour les ressortissants de pays tiers sortants, il n'y aura aucun changement dans les règles de migration familiale pour les ressortissants de pays tiers. Le système deviendra tout simplement aussi restrictif pour les ressortissants de l'UE qu'il l'est actuellement pour les ressortissants de pays tiers. Par exemple, les ressortissants de l'UE souhaitant émigrer au Royaume-Uni pour raison familiale, pour épouser un ressortissant britannique, auront besoin de leur sponsor britannique afin de satisfaire à toutes les exigences, notamment gagner au moins 18600 livres sterling par an. Il s'agit là d'une restriction importante, car près de $42 \%$ des actifs britanniques n'ont pas de revenus suffisamment élevés pour atteindre ce seuil9. Si nous y incluons les inactifs, la proportion s'élève à $62 \%$.
Les changements suggérés par le Livre blanc risquent d'avoir des conséquences majeures sur les dynamiques migratoires entre l'UE et le Royaume-Uni au cours des prochaines décennies. Une grande incertitude persiste quant à l'effectivité de ces changements dans le climat politique actuel. Le Livre blanc pose ainsi les fondements de nouveauxflux migratoires, dont les conséquences ultimes dépendent de décisions à venir.

Article traduit de l'anglais par Vanessa Kientz.

9. Madeleine Sumption, Carlos Vargas-Silva, «Love is not all you need : Income requirement for visa sponsorship of foreign family members », op. cit., p. 62. 